\title{
Cesarean delivery under local anesthesia: a literature review
}

\begin{abstract}
Background:Pregnancy oversight and the childbirth process have been modernized with advances in medicine, which have diverged from the natural birthing process. Today many more women are opting for elective caesarean delivery (CD) to reduce the mental, physical, and painful burden of giving birth. In response to patient requests, cesarean delivery birthing procedures are now being performed around the world. In March 2017, the Centers for Disease Control (CDC) reported $32.0 \%$ of pregnancies were delivered via cesarean delivery in the U.S. Recently; there has been a focus on evaluating the use of infiltrative anesthesia during cesarean delivery. Previously infiltrative anesthesia was only considered for use in rare sittings and high-risk patients, in which general anesthesia was not readily available or contraindicated. This article focuses on the rare use of infiltrative anesthesia for cesarean delivery.
\end{abstract}

Discussion:In cases of life threatening high-risk emergency, cesarean delivery is the standard treatment. In some emergency situations or when vaginal delivery is contraindicated, barriers exist towards administration of general or regional anesthesia. A review of the literature identifies historic reports of an alternative pain management, in such scenarios. Infiltrative anesthesia for cesarean delivery has been previously used in areas where health care funds, hospital resources, and staff are limited, typically in small hospitals and rural communities.

Conclusion: Cesarean delivery under infiltrative anesthesia may be seen as an antiquated method, but it is an important clinical option as it may still have some useful applications. Cesarean delivery under infiltrative anesthesia should be viewed as an alternative in specific situations and not simply a procedure of historic interest.

Keywords: anesthesia, cesarean delivery, cesarean delivery under local anesthesia, infiltration anesthesia, infiltration block, local anesthesia
Volume 9 Issue 3 - 2018

\author{
Shadi Rezai,' Neil D Patel, ${ }^{2}$ Alexander C \\ Hughes, ${ }^{2}$ Ray Mercado, ${ }^{4}$ Takeko Takeshige, ${ }^{5}$ \\ Paul N Fuller,' Cassandra E Henderson ${ }^{3}$ \\ 'Department of Obstetrics and Gynecology, Southern California \\ Kaiser Permanente, USA \\ ${ }^{2}$ St George's University, School of Medicine, Grenada \\ ${ }^{3}$ Maternal Fetal Medicine, Department of Obstetrics and \\ Gynecology, Lincoln Medical and Mental Health Center, USA \\ ${ }^{4}$ Department of Obstetrics and Gynecology, Comprehensive \\ Medical Care of the Bronx, USA \\ ${ }^{5}$ Takeshige Medical, USA
}

Correspondence: Shadi Rezai MD, Department of Obstetrics and Gynecology, Southern California Kaiser Permanente, Kern County, 1200 Discovery Drive, Bakersfield, California, 93309, USA,Tel 7I85795000, Email Cassandra.henderson@nychhc.org

Received: March 18, 2018| Published: May 15, 2018

\section{Background}

Pregnancy and childbirth management have been modernized with advances in medicine, often diverging from the natural birthing process. Today many more women around the world are opting for elective caesarean sections (CD). In March 2017, the Centers for Disease Control (CDC) reported $32.0 \%$ of pregnancies were delivered via cesarean delivery in the U.S. ${ }^{1}$ Because cesarean delivery is effective at reducing the mental, physical (pelvic damage), and the painful burden of giving birth, many women prefer cesarean delivery.

In the U.S. various forms of anesthesia are approved for cesarean delivery. Accepted forms include spinal anesthesia, spinal epidural, epidural block, and general anesthesia. ${ }^{1}$ Most commonly, regional anesthesia is used for planned cesarean delivery births, and general anesthesia is often used in emergent cesarean delivery or postcesarean delivery uterine closure and abdominal suturing. ${ }^{2}$ Regional anesthesia, spinal or epidural, allows the patient to remain conscious throughout the procedure. As with many other medical procedures, there are risks and benefits to each mode of anesthesia. Currently, many providers prefer the use of regional anesthesia because it allows the mother to remain awake throughout the procedure and is safer compared to general anesthesia. ${ }^{2}$

The use of general anesthesia is more common in invasive procedures, mainly for patient comfort and compliance. However, many procedures are still performed using local anesthetic, which primarily functions by hyperpolarizing nerves to block signal transmission. The use of local anesthetic in cesarean delivery (infiltrative anesthesia) involves the anesthetic agent being applied to the subdermal layer, penetrating the various layers of fascia, muscle, and peritoneum, excluding the fatty tissue.$^{3-5}$ Each subsequent layer must be infiltrated once the previous layer has been dissected. ${ }^{4,5}$ As previously known, the use of local anesthetic onset is much slower than that compared to general or spinal anesthesia. Lidocaine has shown to have the quickest infiltrative onset of 10 to 20minutes, compared to other local agents. ${ }^{4,6}$ Compared to general and spinal anesthesia, 10 to 20 minutes is a very long time. However, when evaluating its use for cesarean section with limited resources; it serves as a very useful option, even though onset is longer. Various small incisions can be made to introduce infiltration to ensure adequate block prior to minimize onset time. In relation to the overall procedure, infiltration should be established as quickly as possible to proceed with the cesarean section; especially in emergent cases.

In addition to its use during a cesarean delivery, infiltrative anesthesia for postoperative cesarean care is associated with better outcomes for pain management. ${ }^{7}$ Infiltrating local anesthetic (Bupivacaine or ropivacaine) into the wound has been associated with significant lower opiate (morphine) medication consumption, along with decreased nausea.?

Recently, there has been a focus on evaluating the elective use of infiltrative anesthesia during cesarean delivery. Previously infiltrative anesthesia was only considered for use in rare sittings involving high- 
risk emergent cesarean delivery patients, in which general or regional anesthesia was not readily available or contraindicated - particularly in obese patients, patients who have difficult airway management, or severe coagulopathy. ${ }^{1,3,4}$ This article focuses on the rare use of infiltrative anesthesia for cesarean delivery: assessing the overall outcome of the procedure, in relation to mother and infant and postoperative pain management.

\section{Discussions}

In cases of life threatening or high-risk pregnancies, cesarean delivery is often the mode of delivery. However, in some highrisk and emergency situations, or in cases of patients with medical contraindications, cesarean delivery using regional or general anesthesia cannot be performed. Revisiting a method form the past provides an alternative in such scenarios. The use of infiltrative anesthesia for cesarean delivery has been utilized in areas where a provision of adequate patient care is strained by limited funds, resources and staff in small hospitals or rural communities. ${ }^{8-10}$ Meanwhile, in the past, clinicians have successfully used this technique simply because it was the least complicated, providing rapid anesthesia without modifying the maternal-fetal pathophysiology. ${ }^{5}$

Our current advancements in pain management and invasive operative procedures have distorted our view of the effectiveness of infiltrative anesthesia, which is now considered to be regressive. However, evidence suggests that it may still be a life-saving option for high-risk pregnant women and their offspring. ${ }^{10} \mathrm{~A}$ few fetal highrisk cases include fetal asphyxia, prematurity, maternal diabetes, and Rh-immunization. ${ }^{11}$ The procedure became a standard of practice in regions of Africa, specifically Nigeria, as well as cases being reported in India (1996) and the United Kingdom (1999). ${ }^{12,4}$ In contrast, the use of infiltrative anesthesia for cesarean delivery is seldom seen in modern day American healthcare practices.

\section{Description of procedure and medications}

Cesarean delivery must be performed in a delicate manner, keeping in mind that the mother is fully awake throughout the procedure, and it has been suggested that a professional support person be present in order to prevent patient distress. ${ }^{4}$ Table 1 outlines the potential anesthetic agents which can be used; however, Lidocaine is considered the medication of choice, because it has a rapid onset with the fewest neonatal neurobehavioral side effect. ${ }^{4}$ Table 2 demonstrates the stepwise procedure of infiltrative anesthesia induction during cesarean delivery. Initial placement of local anesthesia is performed using the common wheel pattern at the umbilicus. ${ }^{4}$ Anesthesia is infiltrated through each peritoneal layer prior to incision; although $10 \mathrm{mcg}$ of fentanyl intravenously may be delivered for additional pain relief, in addition to Entonox delivered via face mask. ${ }^{4}$

Unlike standard delivery by cesarean delivery, there are surgical limitations depending on the clinical setting and obstetrical conditions. Even though the procedure may be started using infiltrative anesthesia, completion of the procedure during an emergent delivery may result in use of general anesthesia for uterine closure and abdominal suturing. ${ }^{4}$

In certain settings there is a concern for maintaining hydration and fluid status, along with establishment of an intravenous line to deliver systemic medication. In emergency scenarios, it can be difficult to establish normal intravenous access, due to various circumstances venous collapse, brittle veins, obesity, edema, intravenous infiltration. An alternative, mainly used in children, who can be applied in adults, is rapid establishment of intraosseous infusion (IO). Intraosseous access quickly allows for rapid fluid resuscitation and medication delivery through direct injection into the bone marrow. ${ }^{13,14}$ Intraosseous access provides a more stable, non-collapsible entry point into the venous system; however, precaution should be taken to evaluate the injection site to prevent fluid accumulation in the leg cavity, resulting in compartment syndrome. ${ }^{13,14}$ In addition, intraosseous access allows for ease of pain management techniques when regional or general anesthesia cannot be initiated; particularly in this presentation for infiltration anesthesia cesarean delivery.

Table I Recommended Maximum Dosages for Local Anesthetic Agents. ${ }^{4}$

\begin{tabular}{|c|c|c|c|}
\hline Agent used & Epinephrine & Percentage & Maximum dose*十 \\
\hline Prilocaine $e^{4,6}$ & Without & 0.5 & $80 \mathrm{~mL}(400 \mathrm{mg})$ \\
\hline Prilocaine & With & 0.5 & $120 \mathrm{~mL}(600 \mathrm{mg})$ \\
\hline Mepivacaine & Without & 0.5 & $40 \mathrm{~mL}(200 \mathrm{mg})$ \\
\hline Mepivacaine & With & 0.5 & $80 \mathrm{~mL}(400 \mathrm{mg})$ \\
\hline Lidocaine $\neq$ & Without & 0.5 & 40mL (200mg) \\
\hline Lidocaine ${ }^{4,6}$ & With & 0.5 & $100 \mathrm{~mL}(500 \mathrm{mg})$ \\
\hline
\end{tabular}

*Doses should be halved when the $1 \%$ solution is used.

†Epinephrine must be used in a dilution of $\mathrm{I}: 200,000$ or greater. Side effects may include pallor, sweating, tachycardia, hypertension, and ventricular arrhythmias including ventricular fibrillation. Advantages of using a vasoconstrictor include prolongation of local anesthetic effect, decreased speed of absorption, and decreased systemic absorption and toxicity.

łLidocaine is recommended because of its rapid onset ( 10 to 20 minutes) and least effect on neonatal neurobehavioral reflexes.

Table 2 Local Infiltration Anesthesia for Cesarean delivery. ${ }^{4,6 * T o t a l}$ dose per infiltration should not exceed $500 \mathrm{mg}$

\begin{tabular}{|c|c|}
\hline S. no & Step-wise procedure of infiltrative anesthesia induction \\
\hline $\mathrm{I}$ & Professional support personnel with patient \\
\hline 2 & Skin Infiltration with lidocaine $0.5 \%$ \\
\hline 3 & $\begin{array}{l}\text { Intracutaneous injection in the midline from the umbilicus to the } \\
\text { symphysis pubis }\end{array}$ \\
\hline 4 & Subcutaneous injection lidocaine $0.5 \%$ \\
\hline 5 & Incision down to rectus fascia \\
\hline 6 & Rectus fascia blockade lidocaine $0.5 \%$ \\
\hline 7 & Parietal peritoneal infiltration and incision lidocaine $0.5 \%$ \\
\hline 8 & Visceral peritoneal infiltration and incision lidocaine $0.5 \%$ \\
\hline 9 & Broad ligament lidocaine $0.5 \% 6$ \\
\hline 10 & Paracervical injection lidocaine $0.5 \%$ \\
\hline II & Uterine incision and delivery \\
\hline
\end{tabular}

*Total dose per infiltration should not exceed $500 \mathrm{mg}$

Administration of general anesthesia for uterine repair and closure if needed and able.

Currently intraosseous access is established using the EZintraosseous drill and line placement. Depending on hospital protocol, certification verification, and training, EZ-intraosseous placement can promptly be established within a matter of minutes. ${ }^{15,16}$ For novice physicians and residents, training programs are available to familiarize and practice the protocol. Standard training for EZ-intraosseous access is estimated to take 2 hours, consisting of a lecture supplemented with hands-on training and multiple practice attempts. ${ }^{15,16}$ Even though some hospitals and institutions mandate a thorough intraosseous access training protocol, it is highly recommended for beginners to familiarize themselves with the equipment, procedure, and practice.

\section{Positive aspects of infiltrative anesthesia for cesarean delivery}

The benefits for use of infiltrative anesthesia vary from patient to patient. One of the primary advantages of infiltrative anesthesia 
stems from its safer use in relation to patient respiratory status: unlike general anesthesia, the use of infiltrative anesthesia does not cause severe respiratory compromise. ${ }^{17}$ In comparison, the pulmonary risks and post-operative respiratory function complications are well known with use of general anesthetic, while the use of regional block is circumstantial and unpredictable in some cases. ${ }^{17}$ Another major concern is the risk of intra and post-operative bleeding. The use of infiltrative anesthesia reduces the risk of bleeding in cesarean delivery and provides better surgical outcomes. ${ }^{8}$ Not only are there fewer bleeding complications, but a study performed in Nigeria — which relied on the practice of infiltrative anesthesia for cesarean delivery, due to financial and regional constraints - has substantiated the belief that infiltrative anesthesia in cesarean delivery provides more precise blood pressure control and fewer procedural blood pressure fluctuations. ${ }^{12}$ The study also affirmed lower maternal and perinatal mortality and morbidly rates in women affected by eclampsia. ${ }^{12}$ Aside from the procedural concerns, infiltrative anesthesia can easily be manipulated for patients with structural complications: a successful cesarean delivery case using infiltrative anesthesia in a patient with severe congenital kyphoscoliosis, pelvic tilt, and dislocated hip, with paraplegia from T10-L1 has been reported. ${ }^{17}$

\section{Negative aspects of infiltrative anesthesia for cesarean delivery}

In evaluation of the risks and benefits, the major drawbacks of using infiltrative anesthesia seem minimal compared to those of general and regional anesthesia. The use of infiltrative anesthesia has not shown to be as effective in patients who are obese and/or uncooperative..$^{18,10}$ The use of infiltrative anesthesia helps block the pain; however, the patient is still able to feel pressure, discomfort, and proprioception related to the procedure, preventing its use when patients are overly anxious or are unable to tolerate..$^{2,4}$ In addition, this local method may limit the surgical procedure, as no packs or retractors should be used, reinforcing the importance of gentleness and avoiding sudden movement. ${ }^{19}$ Another aspect in which infiltrative anesthesia has not shown to be beneficial is in multiparous women who have undergone previous or multiple cesarean procedures, resulting in dense fibrous tissue and adhesions preventing the infiltration of the anesthetic agent. ${ }^{10}$

\section{Local anesthesia alternatives}

Many cultures hold faith to the natural birthing process and ultimately desire vaginal delivery. However, with various complications interrupting the normal birthing process, many patients are referred for cesarean delivery. Alternatives to anesthesiabased cesarean delivery are being evaluated, particularly the use of acupuncture. A Chinese study investigated the use of acupuncture anesthesia compared to epidural anesthesia and local anesthesia in cesarean delivery. ${ }^{20}$ Results demonstrated the blood pressure, pulse rate, and respiration remained uninterrupted during the operation; in addition, blood loss was less so than with the use of epidural or local anesthesia, affirming safe use for mother and fetus. ${ }^{20,21}$

\section{Conclusion}

Cesarean delivery under infiltrative anesthesia may be seen as an old outdated method, but it is important not to forget methods we once used in the past, as they may still have some applications (Table 3). As indicated in the discussion, this method of pain management is a viable option when resources, equipment, medication, and personnel are limited - in addition to patient relevant contraindications for regional and general anesthesia. Cesarean delivery under infiltrative anesthesia should be viewed as a safe alternative option, rather than an outdated method for both mother and fetus.

Table 3 Indications for Cesarean under infiltrative anesthesia

\begin{tabular}{ll}
\hline S. no & Indications for cesarean \\
\hline I & Lack of equipment and resources \\
2 & Lack of personnel \\
3 & Contraindication to general and regional anesthesia \\
4 & Need for rapid direct anesthesia - least complicated \\
5 & Least number of maternal-fetal pathophysiology modification \\
6 & Proper intubation cannot be achieved, if necessary \\
\hline
\end{tabular}

\section{Acknowledgements}

The authors would like to thank Ms. Judith Wilkinson, Medical Librarian at Lincoln Medical and Mental Health Center Science Library for providing the reference articles.

\section{Conflicts of interest}

The Authors did not report any potential conflicts of interest.

\section{References}

1. Martin JA, Hamilton BE, Osterman MJ. Births in the United States, 2015. NCHS Data Brief. 2016;(258):1-8.

2. Committee on Practice Bulletins-Obstetrics. Practice Bulletin No. 177: Obstetric Analgesia and Anesthesia. Obstet Gynecol. 2017;129(4):e73e89.

3. Moir DD. Local anaesthetic techniques in obstetrics. $\mathrm{Br} J$ Anaesth. 1986;58(7):747-59.

4. Nandagopal M. Local Anesthesia for Cesarean Section. Techniques in Regional Anesthesia and Pain Management. 2001;5(1):30-35.

5. Ranney B, Stanage WF. Advantages of local anesthesia for Cesarean section, Obstet Gynecol. 197;45(2):163-7.

6. Russell IF. Anaesthesia for emergency caesarean section. Curr Anaesth Crit Care. 1995;6(4):202-205.

7. Li X, Zhou M, Shi X, et al. Local anaesthetic wound infiltration used for caesarean section pain relief: a meta-analysis, Int J Clin Exp Med. 2015;8(6):10213-24.

8. Barker A, Barker M. Caesarean section under local analgesia, Trop Doct. 1976;6(1):23-25.

9. Mahawar B, Baduni N, Bansal P. Cesarean Section under local anesthesia: A step forward or backward? J Anaesthesiol Clin Pharmacol. 2014;30(4):578-9.

10. Shinde G, Sharma N, Jadhav B, et al. Caesarean section under local anaesthesia: back to basics. Trop Doct. 2012;42(1):38-40.

11. Teramo K, Kivalo I, Tarkkanen U, et al. Caesarean section under local anaesthesia for high-risk fetuses. Lancet. 1971;297(7700):646.

12. Fyneface-Ogan S, Uzoigwe SA. Caesarean section outcome in eclamptic patients: a comparison of infiltration and general anaesthesia. West Afr J Med. 2008;27(4):250-4.

13. Leidel BA, Kirchhoff C, Bogner V, et al. Is the intraosseous access route fast and efficacious compared to conventional central venous catheterization in adult patients under resuscitation in the emergency department? A prospective observational pilot study. Patient Saf Surg. 2009;3(1):24.

14. Petitpas F, Guenezan J, Vendeuvre T, et al. Use of intra-osseous access in adults: a systematic review. Crit Care. 2016;20:102. 
15. Anderson TE, Arthur K, Kleinman M, et al. Intraosseous infusion: success of a standardized regional training program for prehospital advanced life support providers. Ann Emerg Med. 1994;23(1):52-5.

16. Ngo AS, Oh JJ, Chen Y, et al. Intraosseous vascular access in adults using the EZ-intraosseous in an emergency department, Int J Emerg Med. 2009;2(3):155-60.

17. Cooper MG, Feeney EM, Joseph M, et al. Local anaesthetic infiltration for caesarean section. Anaesth Intensive Care. 1989;17(2):198-201.

18. Gautam PL, Kathuria S, Kaul TK. Infiltration block for caesarean section in a morbidly obese parturient. Acta Anaesthesiol Scand. 1999;43(5):580-1.
19. Larsen JV, Barker A, Barker M, et al. A technique combining neuroleptanalgesia with local analgesia for caesarean section. S Afr Med J. 1971;45(27):750-1.

20. Wang DW, Jin YH. Present status of Cesarean section under acupuncture anesthesia in China. Fukushima J Med Sci. 1989;35(2):45-52.

21. Bamigboye AA, Hofmeyr GJ. Caesarean section wound infiltration with local anaesthesia for postoperative pain relief - any benefit? S Afr Med J. 2010;100(5):313-9. 